\title{
On the Benefits of Cooperative Video Broadcast over WMANs and WLANs
}

\author{
Yi Liu \\ School of Computing Science \\ Simon Fraser University \\ Surrey, BC, Canada
}

\author{
Cheng-Hsin Hsu \\ School of Computing Science \\ Simon Fraser University \\ Surrey, BC, Canada
}

\author{
Mohamed Hefeeda \\ School of Computing Science \\ Simon Fraser University \\ Surrey, BC, Canada
}

\begin{abstract}
We study the problem of broadcasting video streams over a WMAN to many mobile devices. We propose to form a cooperative network among mobile devices that receive the same video stream, and share received video data over a WLAN. We analytically show that the proposed system outperforms current systems in terms of energy consumption and channel switching delay. Our trace-based simulation results show that the proposed system: (i) achieves as high as $70 \%$ of energy saving gain, (ii) outperforms current systems with only two cooperative mobile devices, (iii) reduce channel switching delay by up to $98 \%$, (iv) is robust under device failure and quickly reacts to network dynamics, and (v) uniformly distributes the load on all cooperative devices.
\end{abstract}

\section{Categories and Subject Descriptors}

C.2.1 [Computer-Communication Networks]: Network Architecture and Design-Wireless Communication

\section{General Terms}

Design

\section{INTRODUCTION}

Video streaming to mobile devices is getting increasingly popular, as mobile devices become powerful and can receive, decode, and display videos sent over networks. In Wireless Metropolitan Area Networks (WMANs), a base station can broadcast videos to support a large number of mobile devices within its coverage range. Streaming videos over WMANs is promising as it supports more mobile devices with lower cost on network infrastructure. Streaming videos to mobile devices is challenging, because mobile devices are battery powered and have stringent energy constraints. Therefore, energy consumption is critical for user satisfaction, because higher energy consumption results in shorter watch time. Several mechanisms have been proposed for video streaming systems to save energy on mobile devices. One typical approach is to broadcast video streams in bursts over WMANs. Mobile devices can then turn off their WMAN interfaces between any two bursts in

Permission to make digital or hard copies of all or part of this work for personal or classroom use is granted without fee provided that copies are not made or distributed for profit or commercial advantage and that copies bear this notice and the full citation on the first page. To copy otherwise, to republish, to post on servers or to redistribute to lists, requires prior specific permission and/or a fee.

MM'09, October 19-24, 2009, Beijing, China.

Copyright 2009 ACM 978-1-60558-608-3/09/10 ...\$10.00. order to save energy. Broadcasting videos over WMANs suffers from high data error rates caused by fading, shadowing, and interference. Since maintaining a large number of reverse connections to the base station for automatic repeat request (ARQ) is not feasible, many WMANs employ forward error correction (FEC) to mitigate the high data error rates.

Current video streaming systems use WMANs to broadcast videos, in which each mobile device independently receives and decodes video data from WMAN base stations. That is, every bit of data from WMANs is used at most once before being discarded. In this paper, we explore the potential of better utilizing the received video data by sharing them among several mobile devices that watch the same video stream. More precisely, we propose a new video streaming system, in which several mobile devices receive and cache video streams broadcast in a WMAN and share the received data over a Wireless Local Area Network (WLAN). Such sharing is possible as WLANs are very popular and many public access points are readily available. We analytically compare this system against the current video broadcast systems that only use WMAN to distribute video streams. We show that the proposed cooperative system achieves better performance from several aspects. First, the energy consumption is reduced, because mobile devices can receive bursts from other mobile devices over the WLAN, which consumes less energy compared to receiving bursts over the WMAN. Second, the delay of mobile devices switching to new video streams can be significantly reduced (almost eliminated), because these mobile devices can request an immediate burst transmission from other devices using unicast. Third, since WLANs have shorter ranges, FECs may not be needed for sending video data over them. This relives mobile devices from manipulating FEC codes and reduces the processing overhead on them.

\section{RELATED WORK}

Several energy saving mechanisms have been proposed in the literature for broadcasting videos over WMANs. For example, in dedicated mobile TV networks, each TV channel is broadcast in bursts at a bit rate much higher than the encoding rate of that TV channel. Mobile devices can then receive a burst of traffic and turn off their radio frequency (RF) circuits until the next burst. This is called time slicing. The authors of [3] study the energy saving achieved by time slicing for any given broadcast scheme. The authors of [1] propose an energy saving strategy by not receiving part of the bursts once the received portions are enough to reconstruct the original data. The authors of [4] consider the problem of constructing broadcast schemes for the base station in order to maximize the energy saving on mobile devices. These works are orthogonal to ours, because they assume all mobile devices receive video data from their WMAN interfaces, while our work considers shar- 


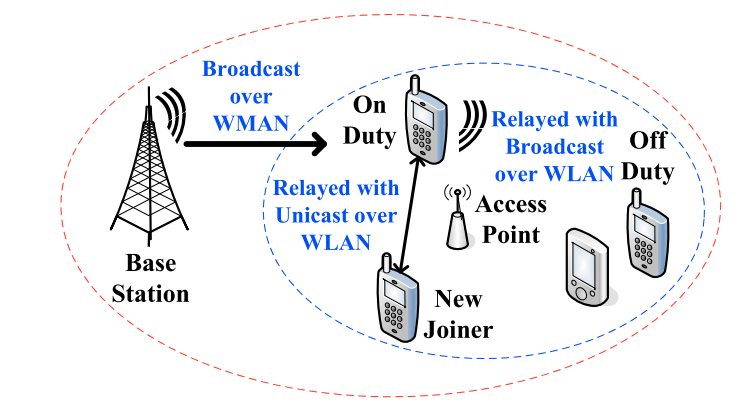

Figure 1: The proposed cooperative video broadcast system.

ing video data over a WLAN in order to save energy beyond what can be saved by time slicing. Channel switching delay refers to the time period between when a user switches to a new video stream until his/her mobile device starts rendering that stream. Channel switching delay consists of several components [6], and time slicing delay is a dominating component among them. Time slicing delay is the time period between the mobile device tunes to the new video stream until the first burst of that video stream arrives. We only consider time slicing delay in this work, and assume other delay components are constant.

\section{OVERVIEW OF PROPOSED SYSTEM}

\subsection{System Architecture}

We consider a video broadcast system with many mobile devices as receivers, where each mobile device joins a WMAN and a WLAN as illustrated in Fig. 1. The base station of this WMAN concurrently broadcasts multiple video streams to all mobile devices. These video streams are sent in bursts, and the mobile devices can receive a burst of data and turn off their RF circuits to save energy. While the WMAN covers a much larger area compared to the WLAN, receiving video data from the WMAN may consume more energy than from the WLAN. This is because a WLAN covers fewer mobile devices, and thus is less sensitive to path loss and shadowing from the base station as well as contention and interference among mobile devices. Therefore, broadcasting video streams over the WLAN achieves higher transmission rates than over the WMAN. Transmitting video streams over WLANs at higher rates leads to shorter transmission time, and allows mobile devices to turn off the RF circuits for longer time, thus reduces energy consumption.

To benefit from the higher rates in the WLAN, we propose to form a cooperative network among all mobile devices that are viewing the same video stream. We let $N$ be the size of the cooperative network. We then run a leadership protocol among these $N$ mobile devices to select a device that is on-duty. The on-duty device receives data bursts from the WMAN base station, and relays the data bursts to other mobile devices that do not receive data from the WMAN. We also select $K-1$ backup devices, which monitor the status of the on-duty device and initiate a switch-over if the on-duty device fails or leaves the system. $K$ is a system parameter. All other $N-K$ devices are off-duty mobile devices, which receive the data bursts over the WLAN from the on-duty device. Last, the on-duty device offers the most recent burst to mobile devices that newly joined this cooperative network. This allows new mobile devices to start playing out faster, as they do not need to wait for the next broadcast burst in WMAN.

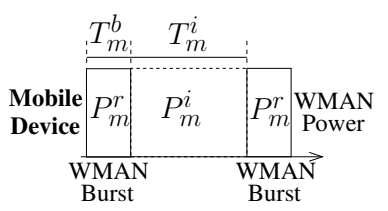

Figure 2: Current system.

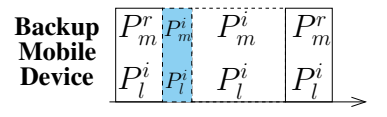

$\underset{\text { Mobile }}{\text { Off-duty }}: P_{m}^{i}\left|P_{m}^{i}\right| P_{m}^{i} \mid P_{m}^{i}$ \begin{tabular}{l:c|c:c:c} 
Mobile & $P_{l}^{i}$ & $P_{l}^{r}$ & $P_{l}^{i}$ & $P_{l}^{i}$ \\
\hline
\end{tabular}

Figure 3: Proposed system.

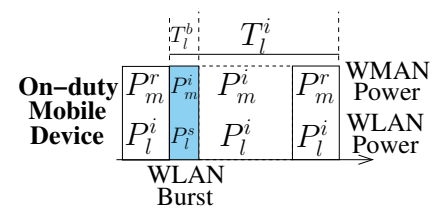

\subsection{Operations}

Burst Relay. The on-duty mobile device relays the received video data bursts to off-duty mobile devices over the WLAN using broadcast. Since the transmission rate of the WLAN is higher than that of the WMAN, receiving video data bursts over WLAN allows off-duty mobile devices to save energy.

Startup Accelerator. Instead of waiting for the next broadcast data burst broadcast on the WMAN, a newly joined mobile device can send a join message to the on-duty mobile device. The on-duty mobile device then transmits the most recent video data burst to the newly joined mobile device using unicast. This allows a device to start playing out immediately after joining the cooperative network.

Electing On-duty and Backup Mobile Devices. Each mobile device in our proposed cooperative system maintains a contribution value, which is the total amount of data that has been received from the WMAN and relayed over the WLAN. Within the proposed cooperative system, an on-duty and $K-1$ backup mobile devices are elected based on the contribution value whenever a video data burst is received. By exchanging multicast message among all mobile devices in the group, the mobile device with the smallest contribution is elected as the on-duty device. Moreover, among other mobile devices, $K-1$ devices with the smallest contribution values are elected as the backup devices.

Fail-safe Mechanism. In the proposed cooperative system, the on-duty mobile devices periodically broadcast alive messages. The backup mobile devices check the status of the on-duty mobile device by monitoring these messages. Once the on-duty mobile device fails, the mobile device with least contribution value among all backup mobile devices takes over and acts as the on-duty mobile device. Since backup mobile devices receive and cache all video data bursts, the video streaming service is not interrupted by the failure of the on-duty mobile device. The off-duty mobile devices turn on their WMAN interfaces if all on-duty and backup mobile devices have failed. The proposed system elects new on-duty and backup devices upon receiving the next video data burst over the WMAN.

\section{ANALYSIS}

Energy Consumption of Current Systems. We first study the energy consumption of mobile devices in current video broadcast systems, in which video streams are sent in bursts over the WMAN. Fig. 2 presents two bursts broadcast over a WMAN to mobile devices. Each mobile device receives a WMAN burst with length of $T_{m}^{b}$ sec, and then puts its WMAN interface into idle mode for $T_{m}^{i}$ sec before reaching the next data burst. The WMAN interface has a receiving power consumption of $P_{m}^{r}$, and an idle power consumption of $P_{m}^{i}$. We compute the energy consumption of all mobile 


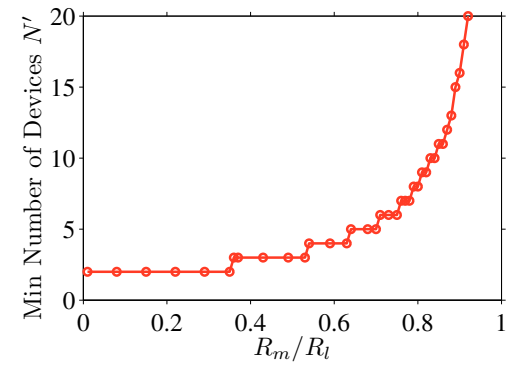

Figure 4: Number of devices to outperform current systems.

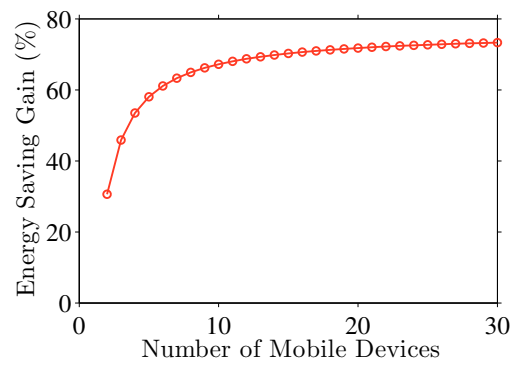

Figure 5: Potential energy saving gain.

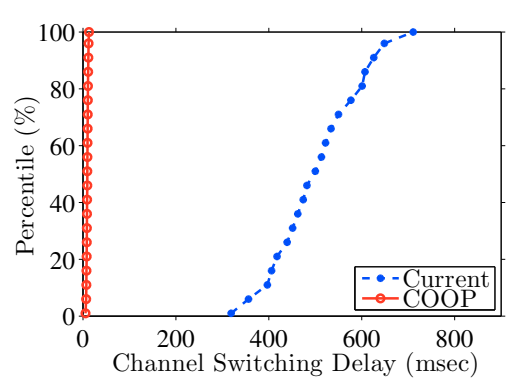

Figure 6: Channel switching delay. devices in the current system between two WMAN bursts as:

$$
E_{e}=N\left(P_{m}^{r} T_{m}^{b}+P_{m}^{i} T_{m}^{i}\right) \text { Joule. }
$$

Energy Consumption of the Proposed System. We study the energy consumption of mobile devices in the proposed cooperative system, where each mobile device has a WMAN interface as well as a WLAN interface. Fig. 3 shows the video data transmission over the WMAN and WLAN. Notice that the main difference between the proposed cooperative system and the current system is that the on-duty mobile device in the former relays the video data burst received from the WMAN over the WLAN to the off-duty mobile devices. These relayed bursts are shaded in Fig. 3. In addition to the aforementioned notations on the WMAN, we let $T_{l}^{b}$ sec be the burst time period and $T_{l}^{i}$ be the idle time period on the WLAN. The WLAN interface has receiving power consumption of $P_{l}^{r}$, sending power consumption of $P_{l}^{s}$, and idle power consumption of $P_{l}^{i}$.

We derive the total energy consumption of the proposed system by accounting for the energy consumption of on-duty, backup, and off-duty devices between two WMAN bursts, and we write it as:

$$
\begin{aligned}
E_{c}= & \left(P_{m}^{r} T_{m}^{b}+P_{m}^{i} T_{m}^{i}+P_{l}^{s} T_{l}^{b}+P_{l}^{i} T_{l}^{i}\right)+ \\
& \left(P_{m}^{r} T_{m}^{b}+P_{m}^{i} T_{m}^{i}+P_{l}^{i} T_{l}^{b}+P_{l}^{i} T_{l}^{i}\right)(K-1)+ \\
& \left(P_{m}^{i} T_{m}^{b}+P_{m}^{i} T_{m}^{i}+P_{l}^{r} T_{l}^{b}+P_{l}^{i} T_{l}^{i}\right)(N-K) .
\end{aligned}
$$

Comparison of Energy Consumption. We derive the sufficient condition for the proposed cooperative system to outperform current systems. Observe that, since the energy consumption of the on-duty device is higher than that of the off-duty device, more devices lead to lower energy consumption. Therefore, we let $N^{\prime}$ be the minimum number of mobile devices for the proposed system to outperform current systems. Combining the Eqs. (2) and (1), and rearranging the inequality (details are not given due to space limitations), we write $N^{\prime}$ as:

$$
N^{\prime}=\left\lceil\frac{\left(P_{l}^{s}-P_{l}^{i}\right) T_{l}^{b}+K\left[\left(P_{m}^{r}-P_{m}^{i}\right) T_{m}^{b}-\left(P_{l}^{r}-P_{l}^{i}\right) T_{l}^{b}\right]}{\left(P_{m}^{r}-P_{m}^{i}\right) T_{m}^{b}-P_{l}^{r} T_{l}^{b}-P_{l}^{i} T_{l}^{i}}\right\rceil .
$$

Note that keeping $N^{\prime}$ small is important, otherwise the proposed cooperative system may lead to worse performance than current systems if there are very few mobile devices in the cooperative network. For illustration, we consider common network parameters and compute the $N^{\prime}$ values under different WMAN and WLAN technologies. More precisely, we vary the $R_{m} / R_{l}$, where $R_{m}$ is the rate of WMAN and $R_{l}$ is the rate of WLAN, and we use Eq. (3) to compute $N^{\prime}$. We plot the results in Fig. 4. This figure shows that $N^{\prime}$ is fairly small. For example, when the WLAN rate is two times faster than the WMAN rate $\left(R_{m} / R_{l}=0.5\right)$, the proposed system only needs 3 devices $\left(N^{\prime}=3\right)$ to outperform current systems.

\section{EVALUATION}

We use trace driven simulations to evaluate the proposed cooperative system. We have implemented several algorithms of the proposed cooperative system, such as the on-duty (backup) device election and fail-safe mechanism, in the simulator. We refer to the proposed system as COOP in the figures. For comparisons, we have also implemented the current system, which only uses the WMAN for broadcast, and we call it Current. For realistic simulations, we have analyzed a transport stream (TS) generated by a Nokia mobile TV base station. This TS consists of four video streams for five minutes, where each channel is encoded at 450 kbps using H.264/AVC video codec and AAC audio codec. We have developed a software utility to analyze the TS and create a log file for each stream. The log files indicate the start time of each burst and its size. We have conducted the simulations with all four video streams. We present sample results of video stream 1 due to space limitations; all other results are similar. Finally, we define the power consumption parameters following the chip data sheets given by the manufacturers as follows. For WMAN power consumption, we consider a mobile TV chip [2], which has receiving power consumption $p_{m}^{r}=400 \mathrm{~mW}$ and idle power consumption $p_{m}^{i}=10 \mathrm{~mW}$. For WLAN power consumption, we consider an 802.11 WLAN chip [5], which has standby power consumption of $p_{l}^{i}=2 \mathrm{~mW}$, receiving power consumption of $p_{l}^{r}=375 \mathrm{~mW}$, and sending power consumption of $p_{l}^{s}=592 \mathrm{~mW}$. We use these parameters in our simulations.

Potential Energy Saving. We first study the potential energy saving that can be achieved by using the proposed cooperative system. We define the energy saving gain as $E_{g}=\left(E_{e}-E_{c}\right) / E_{e}$, where $E_{c}$ is the energy consumption of the proposed cooperative system, and $E_{e}$ is that of current systems. We vary the number of mobile devices from $N=1$ to 30 . For each $N$ value, we compute the average $E_{g}$. We plot the results in Fig. 5. We draw two observations out of this figure. First, the energy saving gain increases as the number of mobile devices increases. Second, the proposed system outperforms the current systems if $n \geq 2$. For example, with only two mobile devices, the proposed cooperative system saves more than 30\% energy compared to current systems.

Eliminating Switching Delay. Next, we study the channel switching delay under user dynamics. To conduct realistic experiments, we develop a software component in our simulator to emulate a large number of channel switching events, and we use it to emulate 100 mobile devices that are viewing one of the four video streams. We generate random channel switching events using Bernoulli trials, and we set the probability of success in a way that users stay with one video stream for $30 \mathrm{sec}$ on average. For each channel switching event, we randomly select a new video stream other than the currently viewed one. We compute the channel switching delay 


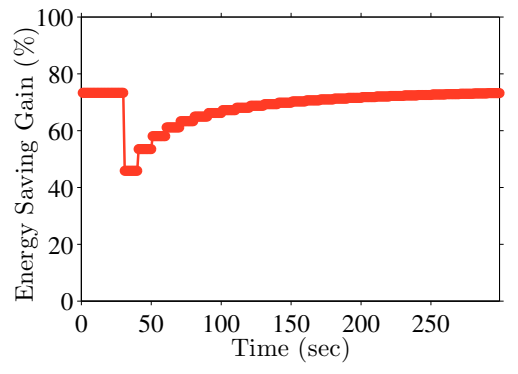

Figure 7: Energy saving under network dynamics.

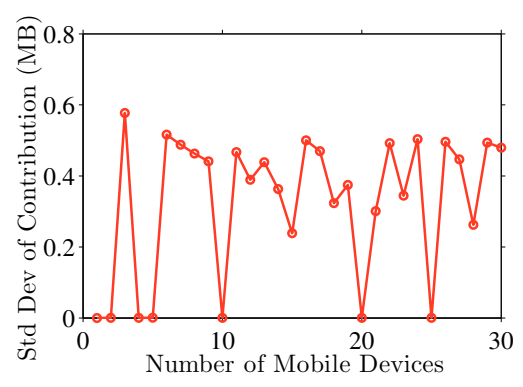

Figure 8: Fairness of the proposed system.

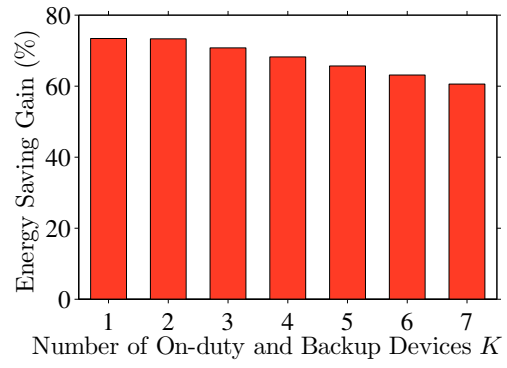

Figure 9: Impact of number of backups on energy saving. by measuring the time difference between when a client decides to switch video stream and gets the data for the new video stream. We then compute the average channel switching delay of each mobile device, and we plot the CDF curves in Fig. 6. This figure illustrates that the proposed cooperative system effectively eliminates the channel switching delay: from up to $700 \mathrm{msec}$ in current systems to at most $13 \mathrm{msec}$ in the proposed system.

Fail-safe Mechanism. We study the effectiveness of the fail-safe mechanisms under mobile device dynamics. We emulate 30 mobile devices that view the same video stream for five minutes. We configure the system to have an on-duty and a backup mobile devices, and instruct the simulator to fail $90 \%$ of the mobile devices after the first $30 \mathrm{sec}$. In addition, we gradually add these failed mobile devices back in order to emulate that the users either reboot their mobile devices or adjust the antennas for better reception. More precisely, we insert a mobile device every ten seconds. We compute the average $E_{g}$, and we plot the results in Fig. 7. We draw two observations out of this figure. First, the proposed cooperative system is robust, because it survives a sudden loss of $90 \%$ of mobile devices, despite under a minor energy saving hit of about $27 \%$. Second, the proposed cooperative system quickly adapts to the network dynamics, because once the failed mobile devices rejoin the cooperative network, the energy saving increases.

Fairness of the Proposed System. Next, we consider the fairness of the proposed cooperative system. We consider an on-duty and a backup mobile devices in this experiment, and we use the simulator to emulate $N$ mobile devices that view the same video stream for five minutes. We vary the value of $N$ from 1 to 30 . We collect the contribution (in terms of number of bytes) of each mobile device. For each $N$ value, we compute the standard deviation of the contribution among all mobile devices, and we plot the results in Fig. 8. This figure shows that the standard deviation of contribution is always less than $0.6 \mathrm{MB}$. The figure clearly shows that the proposed cooperative system evenly balances load on mobile devices, given that the total contribution is in the order of several hundred MBs. Fairness is important to mobile devices because they have stringent energy constraints, and any imbalance in the load may drive users away from the cooperative network.

Number of Backup Mobile Devices. We emulate 30 mobile devices that view the same video stream for five minutes, and we vary the number of backup mobile devices from 1 to 6 . We collect the contribution of all 30 mobile devices and we compute the average $E_{g}$ of all mobile devices, and plot the results in Fig. 9. The figure shows that while more backup devices lead to higher robustness, more backup devices also reduce the energy saving gain achieved by the proposed system. Despite the tradeoff between robustness and energy saving, the energy saving gain achieved by the proposed system does not dramatically drop when the number of backup devices increases. As illustrated in this figure, even when devoting $1 / 5$ of mobile devices to be backups, the energy saving gain is still higher than $60 \%$.

\section{CONCLUSION AND FUTURE WORK}

We have studied the problem of broadcasting multiple video streams over a WMAN to many mobile devices. We have proposed a cooperative system, in which several mobile devices share received video data bursts over a WLAN. The proposed system reduces the energy consumption, significantly reduces the channel switching delay, and reduces the processing overhead. Our trace-driven simulation results have shown that the proposed cooperative system is promising, because it: (i) achieves as high as $70 \%$ of energy saving gain, (ii) outperforms current systems with only two (or more) cooperative mobile devices, (iii) reduces channel switching delay from 700 to $13 \mathrm{msec}$, (iv) is robust under device failure and quickly reacts to network dynamics, and (v) uniformly distributes load on all mobile devices. We are currently implementing the proposed system in a real mobile TV testbed.

\section{REFERENCES}

[1] E. Balaguer, F. Fitzek, O. Olsen, and M. Gade. Performance evaluation of power saving strategies for DVB-H services using adaptive MPE-FEC decoding. In Proc. of IEEE International Symposium on Personal, Indoor and Mobile Radio Communications (PIMRC'05), pages 2221-2226, Berlin, Germany, September 2005.

[2] Evolution of DVB-T front-end receivers through integration, June 2007.

http://www.dibcom.info/Images/Upload/ pdf/whitePaper2_integration_MD_V2.pdf.

[3] Digital Video Broadcasting (DVB); DVB-H implementation guidelines. European Telecommunications Standards Institute (ETSI) Standard EN 102377 Ver. 1.3.1, May 2007.

[4] C. Hsu and M. Hefeeda. Time slicing in mobile TV broadcast networks with arbitrary channel bit rates. In Proc. of IEEE INFOCOM'09, Rio de Janeiro, Brazil, April 2009.

[5] Philips BGW211 low-power 802.11 WLAN chip, April 2005. http: //www.mt-system.ru/documents/bgw211.pdf.

[6] M. Rezaei, I. Bouazizi, and M. Gabbouj. Joint video coding and statistical multiplexing for broadcasting over DVB-H channels. IEEE Transactions on Multimedia, 10(7):1455-1464, December 2008. 